\title{
Multiscale Model of Liver DCE-MRI Towards a Better Understanding of Tumor Complexity
}

\author{
Muriel Mescam, Marek Kretowski, and Johanne Bézy-Wendling*
}

\begin{abstract}
The use of quantitative imaging for the characterization of hepatic tumors in magnetic resonance imaging (MRI) can improve the diagnosis and therefore the treatment of these life-threatening tumors. However, image parameters remain difficult to interpret because they result from a mixture of complex processes related to pathophysiology and to acquisition. These processes occur at variable spatial and temporal scales. We propose a multiscale model of liver dynamic contrast-enhanced (DCE) MRI in order to better understand the tumor complexity in images. Our design couples a model of the organ (tissue and vasculature) with a model of the image acquisition. At the macroscopic scale, vascular trees take a prominent place. Regarding the formation of MRI images, we propose a distributed model of parenchymal biodistribution of extracellular contrast agents. Model parameters can be adapted to simulate the tumor development. The sensitivity of the multiscale model of liver DCE-MRI was studied through observations of the influence of two physiological parameters involved in carcinogenesis (arterial flow and capillary permeability) on its outputs (MRI images at arterial and portal phases). Finally, images were simulated for a set of parameters corresponding to the five stages of hepatocarcinogenesis (from regenerative nodules to poorly differentiated HepatoCellular Carcinoma).
\end{abstract}

Index Terms-Complex system, computational modeling, image analysis, liver tumors, magnetic resonance imaging (MRI) simulation.

\section{INTRODUCTION}

C OMBINING image analysis and computational modeling is one of the possible means to understand complex living systems [1]. The liver, and its pathological modifications, such as tumors, is a system with very complex dynamics, especially related to its original double vascular supply. Understanding the anatomical (structural) and functional processes underlying the observations, whatever the modality, is a necessary step to a better characterization of lesions and consequently to propose the best therapy to the patient. The definition of a model

Manuscript received July 17, 2009; revised August 19, 2009; accepted August 20, 2009. First published September 15, 2009; current version published March 03, 2010. Asterisk indicates corresponding author.

M. Mescam was with INSERM, U642, Rennes, F-35000, France and was also with the Université de Rennes 1, LTSI, Rennes, F-35000, France. She is now with the Department of Physiology and Biotechnology and Bioengineering Center, Medical College of Wisconsin, Milwaukee, WI 53226 USA (e-mail: mmescam@mcw.edu).

M. Kretowski is with the Faculty of Computer Science, Bialystok Technical University, 15-351 Bialystok, Poland (e-mail: m.kretowski@pb.edu.pl).

*J. Bézy-Wendling is with INSERM, U642, Rennes, F-35000, France and also with Université de Rennes 1, LTSI, Rennes, F-35000, France (e-mail: johanne.bezy@univ-rennes1.fr).

Color versions of one or more of the figures in this paper are available online at http://ieeexplore.ieee.org.

Digital Object Identifier 10.1109/TMI.2009.2031435 is always done taking into account the way it will be used. In this paper, we propose a model-based image analysis approach whose objective is to give a physiological sense to parameters extracted from dynamic contrast enhanced magnetic resonance imaging (DCE-MRI) of the liver. These features result from many interacting components associated to organ characteristics (tissue, vascularization) and to image formation parameters (contrast agent, sequence). We focused on the liver and its most widespread malignant tumor, which is the HepatoCellular Carcinoma (HCC). Worldwide, it is one of the most common malignant primitive tumors; its incidence has been regularly increasing over the past years without slowing down [2], [3] and the prognosis is generally bad. Several kinds of therapy, including resection, radio frequency ablation, chemotherapy, and antiangiogenic molecules, can be proposed to patients. Regardless of the therapeutic solution, the treatment will be better if the tumor detection and characterization are realized during the first stages of its development, when the tumor size is less than 2 or $3 \mathrm{~cm}$, and when the hepatic function is still correct. MRI is a noninvasive and very safe observation method, particularly well adapted to evaluate hepatic lesions because it provides a good lesion to liver contrast [4]. The use of contrast agents, and especially those based on gadolinium, provides an estimation of the tumor hypervascularization, and perfusion modifications, which are the main markers of the tumoral growth and malignancy. Rapid acquisition MRI sequences allow us to follow the enhancement just after injection and then to show the differences of biodistribution of the contrast medium between normal and tumoral tissues. However, the correct interpretation of MR images of HCC, and consequently the resulting diagnosis, requires a good comprehension of the physiological phenomena involved in the contrast agent distribution, and of the physical processes related to the image formation. DCE-MRI, based on sequences of MR images acquired after contrast agent injection, is used in clinical practice to assess liver tumor malignancy. Classical pharmacokinetic models are usually fitted to perfusion curves extracted from DCE-MRI, in order to determine physiological markers such as $K_{\text {ep }}$ and $K_{\text {trans }}$, two constants that are used to classify tumors. The contribution of such analysis to the diagnosis and classification of liver lesions has been proven [5] and is not discussed here. However, as far as the comprehension of physiological phenomena is concerned, DCE-MRI is somewhat limited regarding the amount and variety of the available data. Since the technique is based on real data, it is impossible to control every step that leads to the observation (image contrast) from the modifications occurring in the organ, to the image formation. Physiological modeling represents a noninvasive way to control parameters that would be difficult or even impossible to 
Model of hepatic vascular network

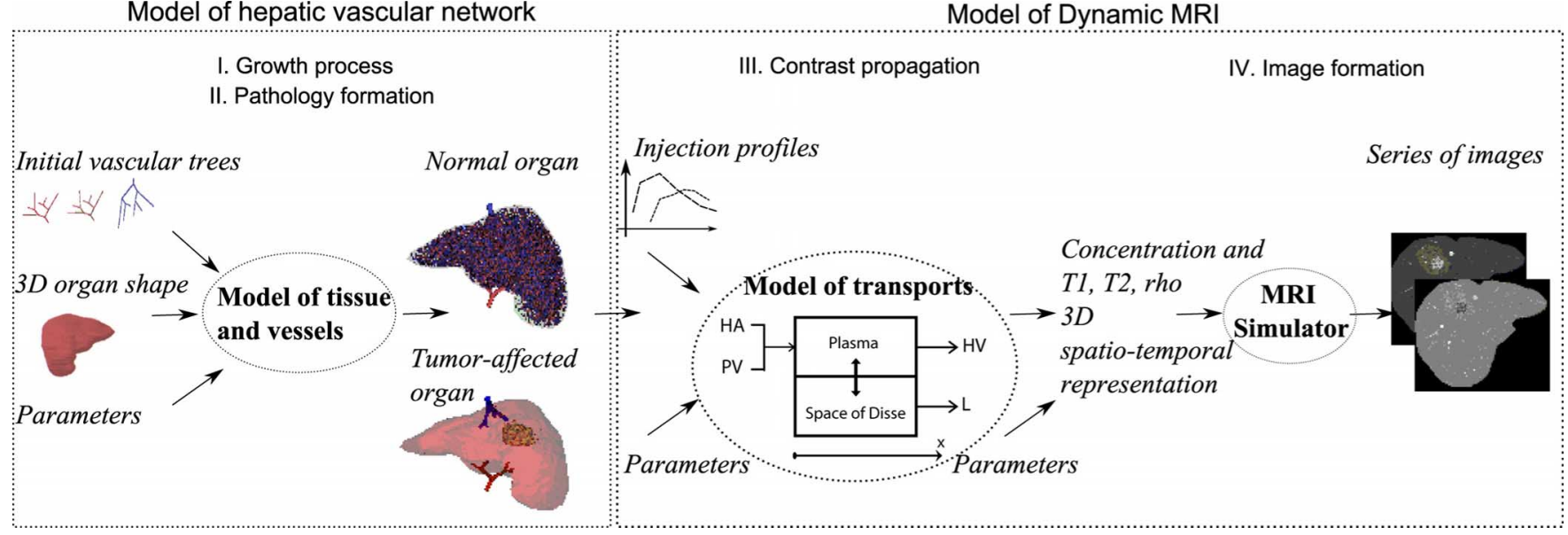

Fig. 1. Coupling of a physiological model of the liver and a model of dynamic MRI acquisition.

access with real experiments. In this aim, we propose the framework presented in Fig. 1, where the coupling between a model of the tumoral liver and a model of dynamic MRI is presented. This multiscale model of liver DCE-MRI provides data we already have a precise knowledge on, in order to relate physiological phenomena to simulated observations. The general aim here is not to replace DCE-MRI but to provide understanding of the mechanisms of production of the DCE-MRI signal, based on a detailed model of contrast agent transport in the liver. Two main steps can be distinguished in this model-based approach: 1) the macroscopic model of the liver, including tissue and vessels, where the structure and the geometry of the vascular network are simulated in normal and tumoral cases (see Section II-A), and 2) the dynamic image formation model including transport of the contrast agent and microscopic characteristics related to this transport at the capillary level (see Section II-B). Only a brief presentation of the macroscopic model is given in the next section, compared to the more detailed description of the dynamic image formation model. Indeed, the most important and original part of the work presented here concerns the model of parenchymal distribution of MRI contrast agents. Detailed explanations on the macroscopic model and some of its applications can be found in our previous studies [6]-[9]. Results are presented in the last section: a sensitivity analysis of the model for two main physiological parameters (blood flow and permeability), and an MRI simulation of HCC in different pathophysiological conditions describing the main steps of carcinogenesis from normal liver to poorly differentiated HCC (HCCp) (Fig. 2).

\section{MOdEL BASED APPROACH}

\section{A. Model of the Hepatic Tumor Growth}

In their very recent paper [10], Lloyd et al. present a generic framework for modeling tumor growth and emphasize that two main components, which are tissue and vascularization, have to be integrated in the model. We already proposed a very comparable framework in 1999 [6]. However, when considering an organ such as the liver, this kind of generic approach must be specified because of the very particular irrigation of this organ and its tumors (double blood supply by the hepatic artery and

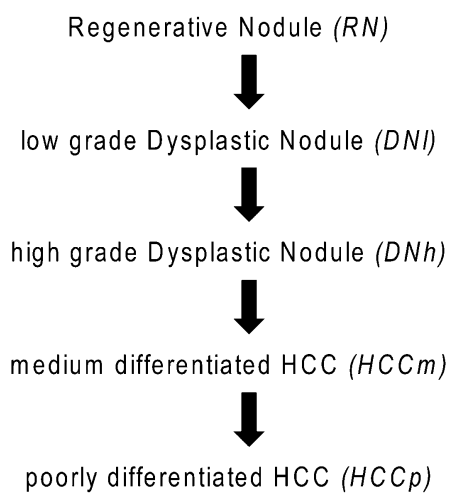

Fig. 2. Main steps of the hepatocarcinogenesis. The best characterization of the tumor (benign nodule, malignant carcinoma) has to be reached in order to propose the most efficient therapy.

the portal vein) and especially if this model is used to simulate dynamic images. This section deals with the macroscopic part of the hepatic computational model. The modeled liver consists of two main components: the tissue (which corresponds to the parenchyma) and the vascular network. The tissue modeling is first explained, taking into account the liver growth, and the possible differences in tissular characteristics. Then, in the vascular model, the structure and geometry of the three hepatic vascular trees (hepatic artery, portal vein and hepatic vein) are precisely considered, as they are essential elements that appear clearly in images and also constitute the pipes that transport blood and contrast agent molecules toward capillaries (place of exchanges between blood and tissue).

1) Tissue Modeling: The tissue is represented by a set of macroscopic functional units (MFU) evenly (but randomly) distributed inside the tridimensional organ shape. Each MFU is a small, fixed size part of the tissue. It is characterized by its spatial, relative position inside the shape, and by its class, which determines most of functional/structural properties (rhythm of mitosis/necrosis), and physiological features (e.g., blood flow rate). Several classes of MFUs can be defined to differentiate functional (or pathological) regions of tissue (normal/tumoral). Furthermore, the MFU class can be modified over time, simulating the possible evolution of a disease (e.g., tumor). Certain 
parameters (such as blood flow rate) of a class are described by their distribution, and are randomly chosen for each new MFU (e.g., this is the case for the terminal blood flow whose natural variability is modeled in such a way).

2) Vascular Network Modeling: A literature review on vascular models is given in [6] and in [11]. Some of them are generic, and others have been specifically developed for a particular organ (such as the heart in [12] and [13] or the kidney in [7]). The tissue is supplied by a vascular network, composed of three trees, with blood going from the arterial and portal trees to the venous one, through the MFUs. A vessel segment (part of vessel between two consecutive bifurcations) is represented by an ideal, rigid tube with fixed radius, wall thickness and position. In the model, all vessels except the capillaries are distinguishable. On the contrary, the geometry of capillaries is not considered, as these smallest vessels are "hidden" in the MFU (see Section II-B1). Based on morphometrical investigation dealing with bigger vessels (e.g., conducted by Zamir and Chee [14]), it is assumed that a single vascular structure has a form of a binary tree. Blood is considered as a Newtonian fluid, with constant viscosity, and its flow is governed by Poiseuille's law (which relates the pressure difference between two extremities of a vessel with the blood flow rate, length and radius of that vessel). At each bifurcation the law of matter preservation is in effect (the quantity of blood, which enters a bifurcation by a vessel, leaves it through its two descendant vessels). Another constraint deals with the decreasing vessel radii in the vascular tree, creating a relationship between the radius of a vessel and radii of its two descendants. Assuming that positions of all vessels are fixed, the vascular trees' consistency is assured. A vascular tree is consistent if: 1) it has the same blood pressure and fixed blood flow in all terminal vessels attached to MFUs and 2) the Poiseuille's law in each vessel, matter preservation and bifurcation laws in each bifurcation are fulfilled. A computationally effective method for the consistency assurance in the vascular tree is described in [15]. Newly appeared MFUs are initially not perfused by the existing vascular network. The closest vessels (in each tree) sprout towards it. A new bifurcation is created, and its geometry is controlled by local minimization of additional blood volume needed for the MFU perfusion. In order to find out the optimal configuration, each candidate vessel temporarily creates a bifurcation perfusing the MFU and the configuration volume is calculated. Additionally, the problem of avoiding possible collisions between perfusing vessels is taken into account. It concerns both intersections of vessels coming from the same tree or from two trees. Finally, the configuration of new bifurcations from all the trees with the lowest sum of volumes is chosen to permanently perfuse the MFU. The next step is devoted to a recalculation of vessels' characteristics (i.e., blood flow, pressures and radii) in the whole tree to fulfill all constraints (i.e., physical and physiological laws). The fast-updating method used to accelerate this time consuming operation is presented in [15]. The development of a pathological process (e.g., tumor) is obtained by means of the conversion mechanism: a conversion represents the period when the current class of MFU inside a chosen region (e.g., sphere) can be changed (with the given probability) to another class. The sequence en-
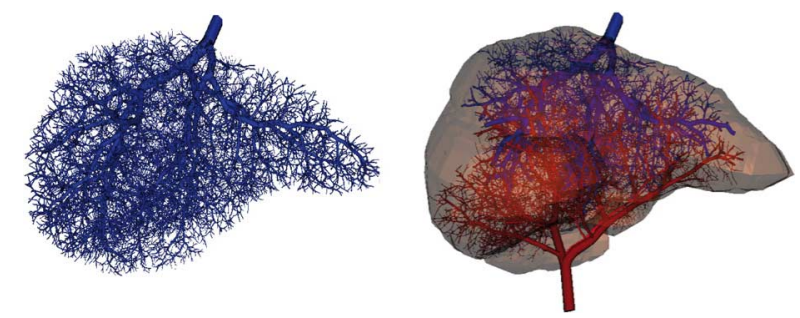

Fig. 3. Hepatic vein is represented until the venule level (smallest vessels have a diameter around 30-50 micrometers). At the end of the growth simulation, the tree counts almost 50000 endings. Right: The three hepatic vascular trees (hepatic artery, portal vein, hepatic vein) are connected. For visualization purposes, the smallest vessels are not represented.

ables to model various stages of pathology development, when macro-cells characteristics evolve gradually.

Some illustration of simulated vascular models can be seen on Fig. 3, where the vascular network of a normal liver is represented.

\section{B. Model of Dynamic MRI}

1) Parenchymal Biodistribution of MRI Contrast Agents: The simulation of the propagation of an MRI contrast agent in the liver parenchyma involves a precise mathematical description of its distribution in every sub-unit of the tissue. As far as gadolinium-based extracellular contrast agents are concerned, the main components to deal with are the plasma region and its surrounding interstitial space-between capillaries and hepatic cells - called the space of Disse. In this aim, a first compartmental Physiologically Based PharmacoKinetic (PBPK) model has been developed and is described in [16]. This five-compartments model considers the terminal branches of the vascular trees (hepatic arteriole, portal venule and hepatic venule) as full compartments, which communicate with the space of Disse. However, since changes in the permeability of these "normally" nonexchanging micro-vessels, are not well defined today, especially in the context of hepatocarcinogenesis, these micro-vessels are assumed impermeable in the present study. As a matter of fact, they only constitute the double arterio-portal input and the hepatic output of the microscopic model. The capillary network (sinusoids) is consequently the only media where exchanges occur, through its fenestrated endothelium (Fig. 4).

These transcapillary exchanges, occurring through the pores and fenestrations of the endothelial cells, are described by a pore model. From physico-chemical parameters related to the targeted molecule (radius $r$ and molecular diffusion $D$ ), and physical parameters related to the capillary membrane (pore radius $r_{k}$ and density $d_{k}$ ), this model enables the computation of transport parameters, such as hydraulic conductivity $L_{k}(1)$, permeability $P_{k}(2)$, and reflection coefficient $\sigma_{k}$ (3). Three types of ideally cylindrical pores, $k$, are considered: small pores $s p$, large pores $l p$, and fenestrations $f$

$$
L_{k}=\left(\frac{A_{k}}{S_{p} \Delta r}\right) \frac{r_{k}^{2}}{8 \eta}
$$




$$
\begin{aligned}
P_{k} & =\left(\frac{A_{k}}{S_{p} \Delta r}\right) D \phi_{k} F\left(\alpha_{k}\right)\left[1+9 \alpha_{k}^{5.5}\left(1-\alpha_{k}^{5}\right)^{0.02}\right] \\
\sigma_{k} & =1-\left[1-\left(1-\phi_{k}\right)^{2}\right] G\left(\alpha_{k}\right)+2 \alpha_{k}^{2} \phi_{k} F\left(\alpha_{k}\right)
\end{aligned}
$$

where $S_{p}$ defines the capillary surface area, whereas the surface area of exchange, $A_{k}$, is obtained from the size and density of the pore $k$ and from $S_{p} . \eta$ is the perfusate viscosity. $F$ and $G$ are two decreasing hydrodynamic functions defined by Curry [17] and recently modified by Bassingthwaighte [18]. These functions depend on the molecule/pore radii ratio $\alpha\left(\alpha_{k}=r / r_{k}\right)$. The solute partition coefficient is defined by $\phi=(1-\alpha)^{2}$.

Transmembranar exchanges are described by Kedem-Katchalsky equations [19], [20], which traduce fluid $\left(J_{v}^{k}\right)$, and solute $\left(J_{s}^{k}\right)$ exchanges for each type of pathway $k$. Both mechanisms (solute and solvant) can be coupled, especially in the case of transport through pores and fenestrations. Fluid movement, based on filtration through the vessel wall, is governed by Starling's hypothesis, and depends on hydrostatic $(p)$ and osmotic ( $\Pi)$ pressure gradients through the membrane

$$
J_{v}^{k}=L_{k}\left\{\left(p_{p}-p_{d}\right)-\left[\sigma_{k}\left(\Pi_{p}-\Pi_{d}\right)\right]\right\}
$$

where indexes $p$ and $d$ stand for the plasma and the space of Disse, respectively. Transport of molecules through the pores of the capillary wall, is described by (5), derived from Patlak's equation [21] and which accounts for concentration gradient through the wall

$$
J_{s}^{k}=P_{k}\left(C_{p}-C_{d}\right) \frac{P e_{k}}{e^{P e_{k}}-1}+J_{v}^{k}\left(1-\sigma_{k}\right) C_{p}
$$

where the Péclet number, $\mathrm{Pe}$, is a dimensionless number measuring the ratio of transport by convection to transport by diffusion. The total fluid and solute fluxes, respectively $J_{v}$ and $J_{s}$, through the sinusoidal membrane, are thus given by the sum of fluxes through each kind of pathway $k$

$$
J_{v, s}=\sum_{k=\mathrm{sp}, \mathrm{lp}, f} J_{v, s}^{k}
$$

One important improvement of the present model, compared to the compartmental model presented in [16], relies on the axial distribution of its plasma and interstitial (space of Disse) regions. Such additional feature allows for concentration gradients along the capillary length and, in this sense, gives a better representation of the real transport processes, than compartmental models where well-stirred regions are filled instantaneously. The new model, presented in Fig. 4, is then comparable to BTEX units (Blood Tissue EXchange units), defined by Bassingthwaighte et al. in [22], where the convection-permeation-dispersion mechanisms are described in the following system of partial differential equations (PDEs):

$$
\begin{aligned}
\frac{\partial C_{p}}{\partial t}= & -\frac{\left(F_{h a}+F_{p v}\right) L}{V_{p}} \frac{\partial C_{p}}{\partial x} \\
& -\frac{S_{p} J_{s}}{V_{p}}+D_{p} \frac{\partial^{2} C_{p}}{\partial x^{2}} \\
\frac{\partial C_{d}}{\partial t}= & \frac{S_{p} J_{s}}{V_{d}}+D_{d} \frac{\partial^{2} C_{d}}{\partial x^{2}}
\end{aligned}
$$

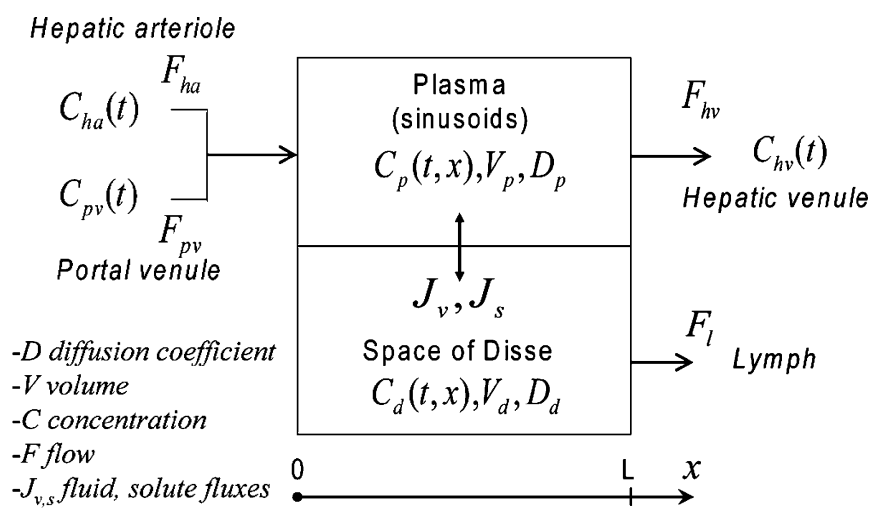

Fig. 4. Axially distributed BTEX model, describing the hepatic microvascular transport of molecules.

where $L$ is the capillary length and $C_{\text {in }}$ is the concentration at the input of the plasma compartment, resulting from the dual arterio-portal supply

$$
C_{\mathrm{in}}=\frac{F_{h a} C_{h a}+F_{p v} C_{p v}}{F_{h a}+F_{p v}} .
$$

In addition, variations in regions' volume have been highlighted in the past by Goresky, with the help of the multiple indicator dilution (MID) technique [23]. In the model, those variations are reflected in pressure variations

$$
\begin{aligned}
& p_{p}=\frac{V_{p}}{\text { Base } \rho} \\
& p_{d}=\frac{V_{d}}{\text { Base } \rho}
\end{aligned}
$$

where Base defines the surface area of the volume base, and enables the conversion from the fluid volume height to a pressure. The choice for the base surface area controls changes in pressure: a small base induces high pressure variations whereas a large base reduces the pressure influence on the flux.

Furthermore, high interstitial pressures $p_{d}$, combined to low lymphatic pressures $p_{l}$, may give rise to an evacuation of the interstial fluid (confined to the space of Disse), via the lymphatic network, thus resulting in a lymphatic outflow $F_{l}$

$$
F_{l}= \begin{cases}K_{L}\left(p_{d}-p_{l}\right), & \text { if } p_{d}>p_{l} \\ 0, & \text { else }\end{cases}
$$

where $K_{L}$ is the lymphatic conductance.

Mass conservation, in this system, is consequently ensured by the following system of ordinary differential equations:

$$
\begin{aligned}
& \frac{d V_{p}}{d t}=-S_{p} J_{v} \\
& \frac{d V_{d}}{d t}=S_{p} J_{v}-F_{l} .
\end{aligned}
$$

Coupling the two levels, in order to obtain a multiscale model of contrast-agent propagation, necessitates the calculation of the global concentration in each functional unit of the macroscopic model (MFU). This is done by (15), from which an averaged concentration is computed

$$
C_{\mathrm{MFU}}(t)=\frac{V_{p} \int_{0}^{L} C_{p}(t, x) d x+V_{d} \int_{0}^{L} C_{d}(t, x) d x}{L\left(V_{p}+V_{d}\right)} .
$$


2) MRI Acquisition Simulation: A 3-D MRI simulator, named SIMRI and recently developed by Benoit-Cattin et al. [24], was used to generate simulated images of the liver. The simulation system is based on the Bloch equation resolution. A 3 -D virtual object constitutes the input of the simulator. Each voxel is defined by three values describing the proton density $\mathrm{PD}$, the spin-lattice relaxation time $\mathrm{T}_{1}$ and the spin-spin relaxation time $\mathrm{T}_{2}$, associated to the corresponding tissue. After application of a MRI sequence on the input, the magnetization kernel computes a set of RF signals, stored in the k-space. Addition of noise to the k-space, associated to its filtering before the reconstruction of the MR image using fast Fourier transform, provides relatively realistic images. Since each voxel of the liver model may contain both types of tissue (blood and parenchyma), the corresponding MRI parameters are computed knowing the proportion of each tissue in the voxel. The generation of new postinjection MRI maps $\left(\mathrm{PD}, \mathrm{T}_{1}\right.$ and $\left.\mathrm{T}_{2}\right)$ is then a necessary step to simulate DCE-MRI. Equation (16) gives the relationship between the relaxation terms $\left(R_{1,2}=1 / T_{1,2}\right)$, characteristic of a voxel, and the associated contrast agent concentration

$$
R_{1,2 \text { postinjection }}=R_{1,2 \text { intrinsic }}+r_{1,2} C(t)
$$

where relaxivities, $r_{1,2}$, are constants that are contrast agent dependent.

\section{SimUlation Results AND DisCUSSION}

\section{A. Simulation Procedure}

All the results presented in this paper were obtained following the same simulation procedure. After coupling both macro- and microscopic models, a virtual tumor is generated, based on a simulated adult liver. Propagation of a Gd-DOTA contrast agent (Dotarem, Guerbet, France), currently used in clinical routine, is simulated, using, as entry functions, real concentration-versustime curves obtained from a DCE-MRI study. The arterial input function (AIF) was measured from a ROI selected in the aorta (arteries are thin and hardly visible on images), on a sequence of MR images acquired after an intravenous bolus injection of Gd-DOTA, with a time step of approximately $10 \mathrm{~s}$. The time needed by the mixing blood-contrast agent to reach the main branch of the hepatic artery is neglected since the aorta-hepatic artery pathway is straightforward compared to the aorta-portal vein pathway. In order to account for the portal delay as accurately as possible, a second ROI was selected in a portal vein (larger than the hepatic artery). Once the agent concentration is computed in every voxel as a function of time, postinjection $\mathrm{T}_{1}, \mathrm{~T}_{2}$, and PD maps are created at typical arterial and portal phases (i.e., when the concentration of contrast agent is first higher in the hepatic artery and then later in the portal vein). Finally, these 3-D maps are used to simulate MR images via the simulator SIMRI. A typical $\mathrm{T}_{1}$-weighted 3-D Gradient Echo sequence is simulated at 3 Tesla (repetition time $\left(T_{R}\right), 2.75 \mathrm{~ms}$, echo time $\left(T_{E}\right), 1.36 \mathrm{~ms}$, flip angle, $\left.10^{\circ}\right)$.

\section{B. Qualitative Analysis}

A HCCp-like virtual tumor is first generated, based on a simulated adult liver. Results presented in Fig. 5(a) correspond to im- a.

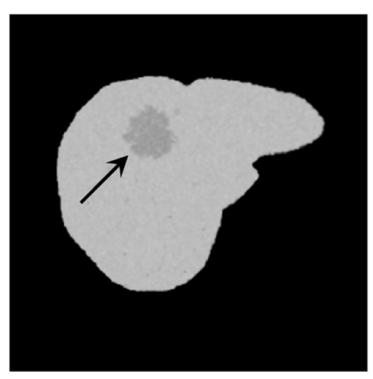

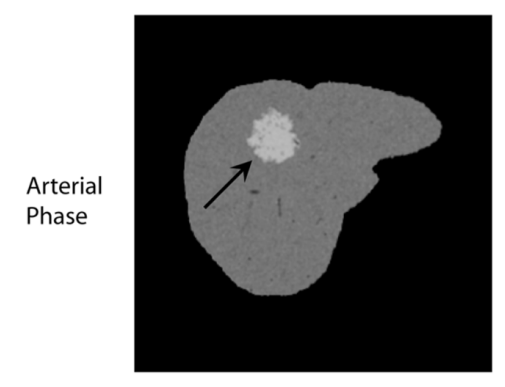

b.
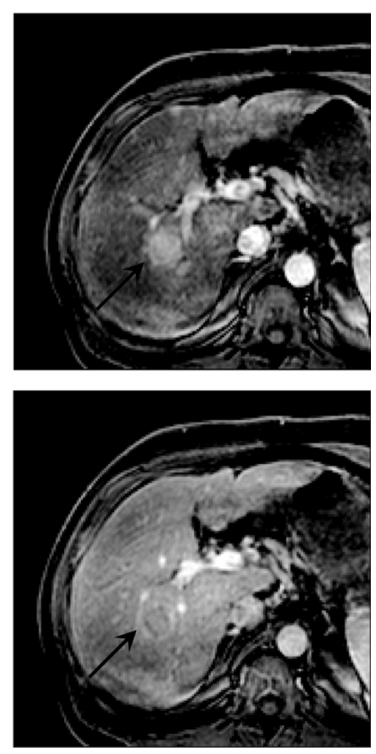

Fig. 5. Comparison between simulated (a) and real (b) dynamic contrast-enhanced MR images of the liver. Acquisition conditions: 3-D Gradient Echo sequence $3 \mathrm{~T}\left(T_{E}=1.36 \mathrm{~ms}, T_{R}=2.75 \mathrm{~ms}\right.$, Flip Angle $=10^{\circ}$, Slice Thickness $=4 \mathrm{~mm}$ ). Contrast agent: Gd-DOTA Top: arterial phase. Bottom: portal phase. Note: arrows indicate the tumor. (a) Simulated MR images of the liver and (b) real MR images of the liver.

ages simulated after applying a $\mathrm{T}_{1}$-weighted $3 \mathrm{D}$ Gradient Echo sequence on a $4 \mathrm{~mm}$-slice. Corresponding real images (obtained from the same acquisition parameters) are presented in Fig. 5(b). At arterial phase, the amount of contrast agent is higher in the tumor than in normal tissue. Indeed, the arterial neovascularization, combined with an inhibition of the portal vascularization, produces an "arterialization" phenomena, known as a typical symptom in hepatic tumoral development. In consequence, the global concentration of Gd-DOTA in the tissue presents a peak that is close to the arterial one. The contrast between tumoral and normal tissues is then higher at this time, and $\mathrm{T}_{1}$ and $\mathrm{T}_{2}$ values, driven by (16), are decreased in the tumor. The $\mathrm{T}_{1}$-weighted gradient echo sequence enhances the $T_{1}$ contrast in images, brightening tissues with lower $\mathrm{T}_{1}$, thus explaining the observed contrast (Fig. 5). Later, at portal phase, Gd-DOTA concentration has decreased and the contrast appears hypointense compared with the normal surrounding tissue (Fig. 5 bottom).

\section{Sensitivity Analysis}

A large number of parameters controls each level of the multiscale model, thus enhancing the difficulty of its identification. However, it is possible and relevant to evaluate its sensitivity to some particular parameters, such as those related to the development of the HCC. For instance, inflows and capillary permeability play an important role in the evolution of the pathology from a benign regenerative nodule to a poorly differentiated HCC (Fig. 2). First, the influence of each parameter is separately evaluated through a sensitivity analysis. The output of the model, i.e., simulated MR images of the liver, is obtained for increasing values of arterial inflow, $F_{h a}$, and then for increasing values of the permeability-surface area product, which is referred to by the notation $P S$ in the following study. In each 


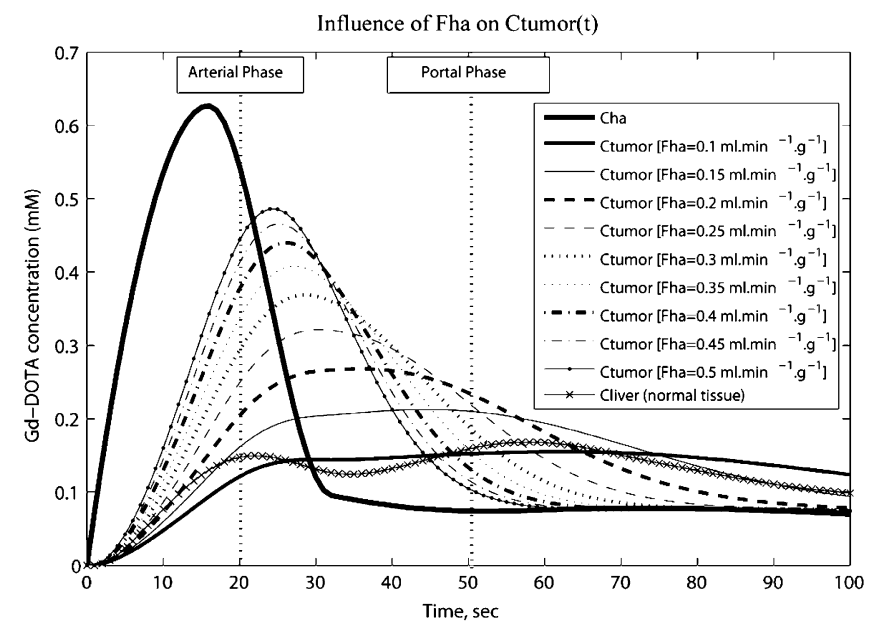

Fig. 6. Simulated Gd-DOTA concentration-versus-time curves in the liver parenchyma, for increasing values of the arterial inflow $F_{h a}$. The parallel evolution of the concentration in the normal tissue is also presented (parameters fixed according to normal conditions). In the tumoral region, $F_{p v}=0$ and the permeability is high.

case, enhancement concentration curves are simulated at the microscopic scale and corresponding simulated bi-phasic (arterial and portal) images are depicted. A "tumoral" region is considered, and the ratio $R_{S}$ of the mean signal in the tumor to the mean signal in the surrounding "normal" tissue is compared to the ratio $R_{C}$ of Gd-DOTA concentration in both tissues.

Concentration-versus-time curves obtained for varying inflows $F_{h a}$, are presented on the graph of Fig. 6. These concentrations are simulated from the distributed model of contrast agent propagation, separately uncoupled from the vascular model, for the classical gadolinium chelate (Gd-DOTA) (15). In order to observe the influence of the arterial inflow only, the portal supply is inhibited, and the parameters controlling the permeability are chosen so that the latter is high and the model is therefore not diffusion-limited. Concentration profiles obtained for increasing arterial flow are well aligned with the established arterialization phenomena that occurs in liver tumors, showing an enhancement peak at the so-called arterial phase. In addition, a nonlinear behavior of the concentration, as a function of $F_{h a}$, can be observed at the portal phase, where the concentration in the liver grows as the inflow grows, to reach a maximum value above which it decreases again. Values of the concentration in the liver, at two particular times after injection, corresponding to arterial (24 s) and portal (50 s) phases, are extracted from these curves, and the ratio $C_{\text {tumoral }} / C_{\text {normal }}$ is depicted as a function of $F_{h a}$, on the right graphs of Fig. 7(a) and (b), respectively. Such representation not only confirms the previous observations regarding the linearity of $C\left(F_{h a}\right)$ at the arterial phase, and its nonlinearity during the portal phase, it also allows expectation of some particular contrast dynamics on simulated MR images. Indeed, a linear relationship exists between the contrast agent concentration and relaxation parameters (16). However, an exact quantification of the contrast enhancement is not straightforward, since the relationship between the tissue relaxations and the effective NMR signal intensity also depends on the applied sequence and
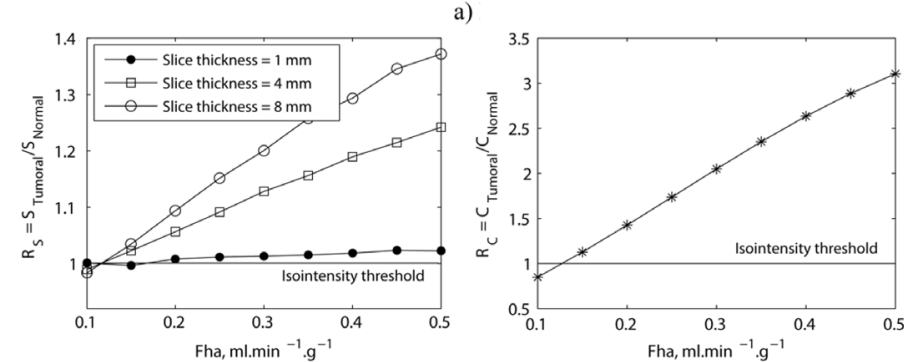

Slice thickness $=4 \mathrm{~mm}$

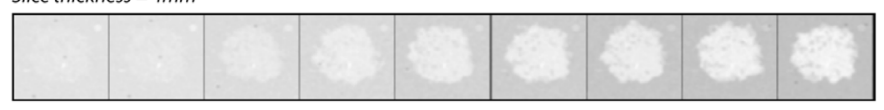

b)
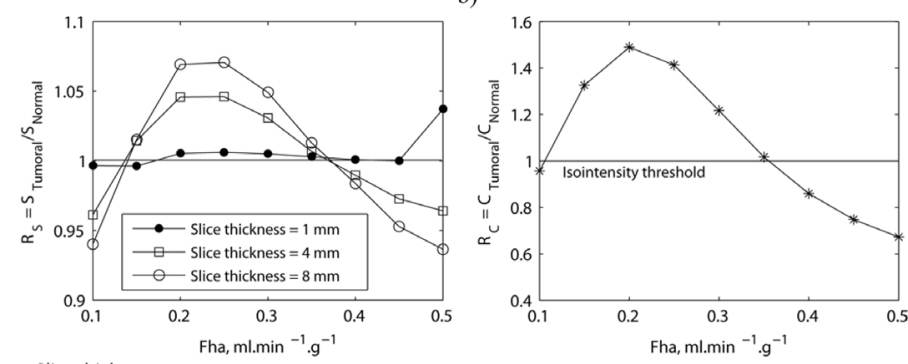

Slice thickness $=4 \mathrm{~mm}$

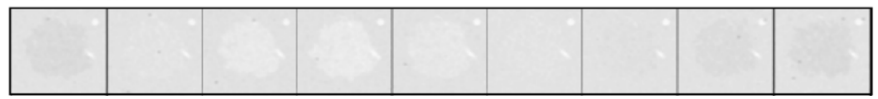

Fig. 7. Evaluation of the influence of the arterial inflow, $F_{h a}$. Its effect on the model is observed at (a) arterial and (b) portal phases, at two different scales: at the macroscopic scale (left-hand graph), the mean signal is measured in two ROIs chosen in the tumoral and in the normal regions, after simulating $\mathrm{T}_{1}$-weighted GRE MR images for three different slice thickness; at the microscopic scale the concentration value is simulated in a "normal" MFU, and a tumoral MFU. Corresponding simulated $64 \times 64 \mathrm{~T}_{1}$-weighted GRE MR images are presented for a $4 \mathrm{~mm}$ slice thickness.

on the acquisition parameters. Nevertheless, contrast dynamics can at least be explained by the concentration dynamics, as shown on Fig. 7(a) and (b), where the behavior of $R_{S}$ with $F_{h a}$ matches that of $R_{C}$. These observations are illustrated by the corresponding set of simulated MR images, on which the contrast tumoral/normal tissue confirms the quantitative measurements. Furthermore, the nonlinearity of the contrast evolution with the inflow at the portal phase, whereas it is linear at the arterial phase, suggests an easier interpretation of real images at the arterial phase, concerning the relationship between the observation on the image and the inflow it reflects. In addition, images were simulated for three different slice thicknesses $(1 \mathrm{~mm}, 4 \mathrm{~mm}$, and $8 \mathrm{~mm})$. From the measurements presented on Fig. 7, it seems evident that the larger the slice, the better the contrast. For very thin slices $(1 \mathrm{~mm})$, it can even become relatively difficult to give a correct interpretation of the relation between the contrast and the inflow. Since the intensity of the signal in the tumor region is higher than in the normal tissue, the SNR increases faster in this region, with the slice thickness, which may explain the higher contrast in this case. In addition, in thicker slices, tumor regions are more vascularized whereas normal tissue is more homogeneous whatever the slice thickness. However, the important issue here is not slice thickness per se but SNR, which can be improved by a number of means other than changing slice thickness, including changing 
the in-plane resolution, changing the receive coil, changing field strength, changing the number of averages.

In the following simulations, the relation between the contrast enhancement and the capillary permeability is studied. This time, results are presented for only one slice thickness. Slices of $4 \mathrm{~mm}$ are a good compromise between the quality of the contrast and the computation time. In addition, this is commonly used in clinical routine, as shown in qualitative observations presented in Section III-B. Among the numerous physiological parameters that control the permeability, variations in the quantity of pores and fenestrations on the membrane of sinusoids are directly related to the capillarization phenomena (progressive disappearance of fenestrations), which is one of the main mechanisms involved in hepatocarcinogenesis. However, it is more common among the modeling community to deal with the permeability-surface area product, $P S$, an upper scale parameter that generally describes solute transport through a membrane barrier. The pore model described in the first section does not allow a direct calculation of $P S$ from the pores' density. As a matter of fact, an estimated value of $P S$ is extracted after fitting concentration curves, simulated for varying values of the densities of small and large pores and fenestrations, to a simple BTEX model in which the diffusion term $S_{p} J_{s}$ is replaced by $P S\left(C_{p}-C_{d}\right)$ in (7) and (8). Simulation results showed a different concentration-time course when setting a low input arterial flow, compared to a high one [Fig. 8(a) and (b)]. Effectively, for a low inflow, the concentration in the liver parenchyma is clearly not dependent on the capillary permeability at both arterial and portal phases and is thus flow-limited. Consequently, almost no variation can be noticed in the signal intensity with this parameter (Fig. 9). In addition, the contrast agent concentration is very close in both classes of tissue, which explains the poor contrast on corresponding simulated images at the two phases. On the contrary, at high inflow, a linear relationship exists between the concentration and the permeability, and then indirectly between the signal intensity and the permeability. This relationship is no longer linear at portal phase. Indeed, when capillaries are weakly permeable, the contrast agent is mainly confined to the vascular space. Consequently, the solute is primarily driven from the arterio-portal entrance to the hepatic venous extremity of the network, by convection and dispersion. The washout is therefore completed much faster. When the permeability is no longer negligible, the diffusion process tends to hinder this washout. However, when it becomes significant, i.e., above some threshold, the steady-state is rapidly reached and the model is no longer diffusion-limited.

Although the previous results show the evidence of the existence of relationships between phenomena observed on images and some physiological parameters that are markers of the tumoral development, the kinetics of contrast enhancement remain difficult to explain. Furthermore, in this sensitivity analysis, only one parameter was varied at a time. However, the real processes involved in the development of a pathology and its evolution are obviously much more complex and imply the variation of a set of parameters instead of a single one. a)

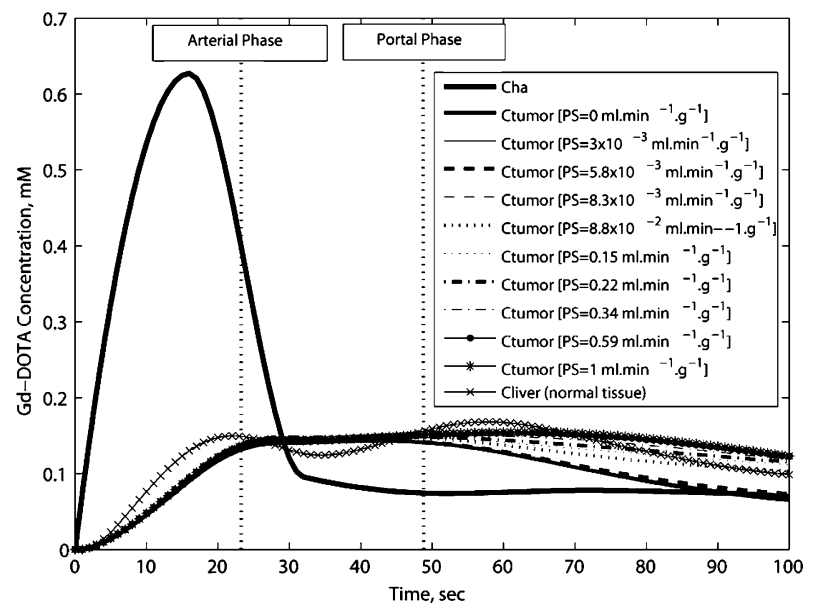

b)

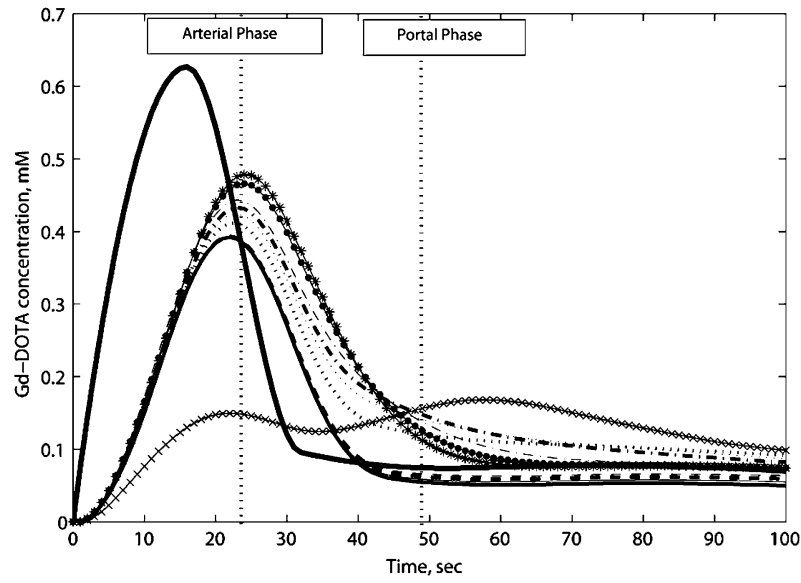

Fig. 8. Simulated Gd-DOTA concentration-versus-time curves in the liver parenchyma, for increasing values of the permeability. The parallel evolution of the concentration in the normal tissue is also presented (parameters fixed according to normal conditions). In the tumoral region, $F_{p v}=0$. Simulation results are given for (a) a low arterial inflow, (b) a high arterial inflow. (a) Influence of PS on Ctumor(t) for low inflow and (b) Influence of PS on Ctumor(t) for high inflow.

\section{Application to the Hepatocarcinogenesis}

The multistep hepatocarcinogenesis describes the different stages of the development of a liver hypervascularized tumor, from benign regenerative nodules (RN) to poorly differentiated hepatocellular carcinoma ( $\mathrm{HCCp}$ ), through respectively lowand high- grade dysplastic nodule (DNl,h), and medium-differentiated $\mathrm{HCC}(\mathrm{HCCm})$ (Fig. 2). Parallel modifications of several physiological characteristics affecting both the tissue and the vascularization are involved in the evolution of the lesion from one step to the other. For instance, we have seen previously that arterial/portal inflows and capillary permeability may affect the observed contrast differently. Gadolinium-enhanced $\mathrm{T}_{1}$-weighted GRE MRI is specifically used to detect HCC because of the highly arterialized neovascularization of this lesion, compared to DNs and RNs. However, the same sequence (same acquisition parameters as used in previous simulations) has been used here to simulate MR images of all different grades, so that only physiological parameters are responsible for differences observed between the simulated images. 
a)

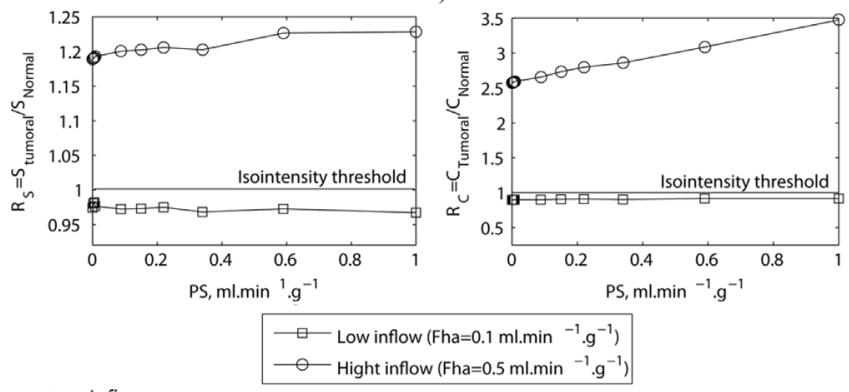

Low infiow

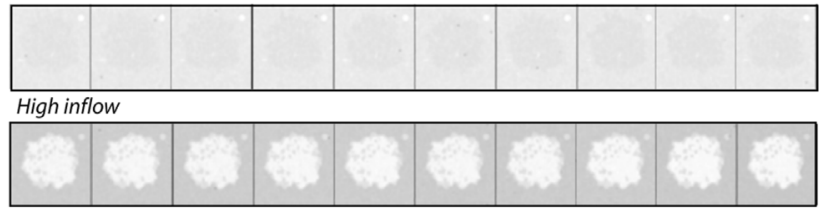

b)
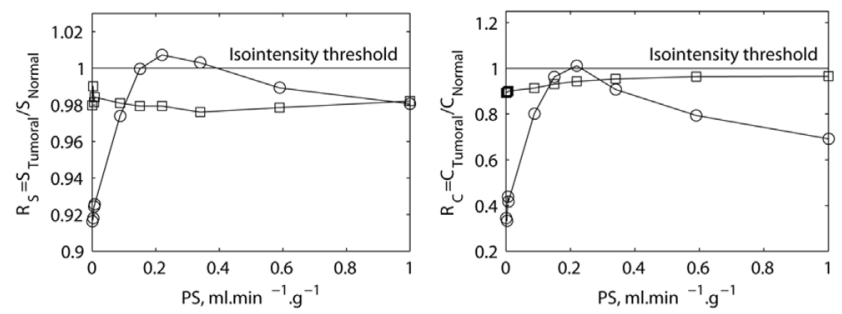

Low inflow

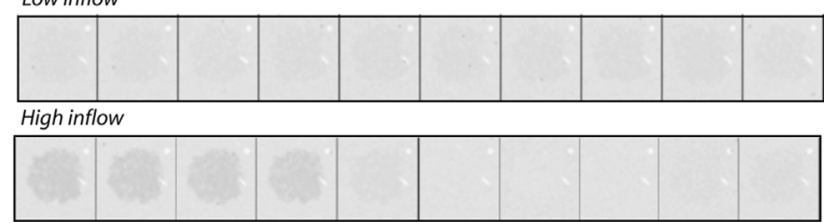

Fig. 9. Evaluation of the influence of the permeability, via the estimated permeability-surface product $P S$. Its effect on the model is observed at (a) arterial and (b) portal phases, at two different scales: at the macroscopic scale (left-hand graph), the mean signal is measured in two ROIs chosen in the tumoral and in the normal regions, after simulating $\mathrm{T}_{1}$-weighted GRE MR images (slice thickness $=4 \mathrm{~mm}$ ); at the microscopic scale the concentration value is simulated in a "normal" MFU, and a tumoral MFU. Corresponding simulated $64 \times 64 \mathrm{~T}_{1}$-weighted GRE MR images are presented for a $4 \mathrm{~mm}$ slice thickness. The model behavior with varying $P S$ is evaluated for two values of the arterial inflow (low and high).

In this section, simulation results are still given at two scales: concentration-time curves at the microscopic scale [Fig. 10(a)], and associated MR images at the macroscopic one [Fig. 10(b)]. This time, though, a set of parameters (arterial, $F_{h a}$, and portal, $F_{p v}$, inflows, and permeability, reflected in the density of fenestrations $d_{f}$ ) is applied in correspondence to the five different stages of hepatocarcinogenesis. The values are given in Table I. Exact values for each grade of the lesion remain undefined and vary according to the study they are extracted from. However, a general behavior of portal and arterial supplies during hepatocarcinogenesis has been established and enables us to define reasonable values [25]. In the present study, arterial and portal flows were thus chosen taking into account normal inflows and relative variations described on the diagram of Fig. 8 in [25]. Similarly, the number of fenestrations is known to decrease progressively in capillary wall so values have been chosen relatively to known normal values [26].
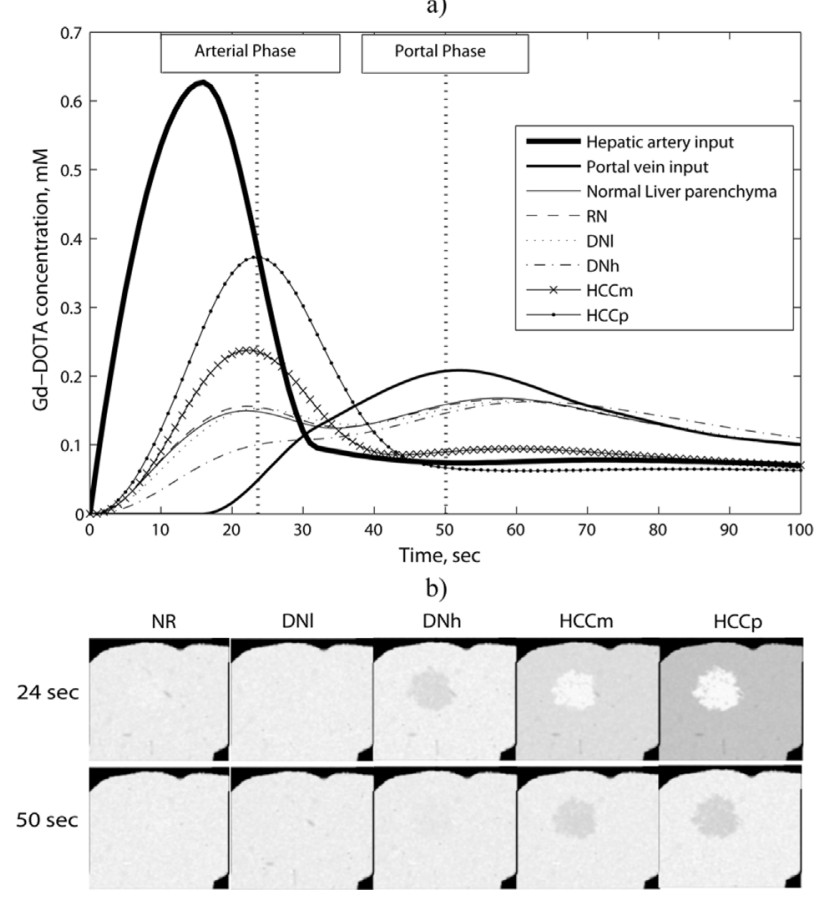

Fig. 10. Evolution of (a) Gd-DOTA concentration profile in the hepatic parenchyma and of (b) $T_{1}$-weighted GRE MR images, with the grade of the lesion during hepatocarcinogenesis. $128 \times 128$ images are simulated from 4 $\mathrm{mm}$ slices. (a) Concentrations at different stages of the hepatocarcinogenesis and (b) corresponding GRE MRI images.

TABLE I

SOME OF THE PHysiological PARAMETERs RElated to EACH StAGE OF THE HEPATOCARCINOGENESIS

\begin{tabular}{|l||c|c|c|c|c|}
\hline lesion grade & NR & DNl & DNh & HCCm & HCCp \\
\hline$F_{h a}\left(\mathrm{ml}^{-1} \mathrm{~g}^{-1} \cdot \mathrm{min}^{-1}\right)$ & 0.27 & 0.2 & 0.1 & 0.3 & 0.4 \\
\hline$F_{p v}\left(\mathrm{ml} . \mathrm{g}^{-1} \cdot \mathrm{min}^{-1}\right)$ & 0.7 & 0.5 & 0.35 & 0.2 & 0 \\
\hline$d_{f}\left(\mathrm{nb} \mathrm{fen} / \mathrm{mm}^{2}\right)$ & 11 & 11 & 5 & 0 & 0 \\
\hline
\end{tabular}

In term of contrast tumor/normal tissue, simulated images are coherent with common observations brought on typical postcontrast $\mathrm{T}_{1}$-weighted (T1W) imaging. For benign lesions such as regenerative nodules (NR) and eventually low grade dysplastic nodules (DNl), arterial and portal inflows are close to normal ones, and sinusoids are still fully permeable, which explains the difficulty, not only in their characterization but also in their detection since there is no arterial enhancement in Gd-DOTA-enhanced T1W images. At early stages of the malignancy, the arterial inflow slightly diminishes, resulting in a hypointense lesion on T1W GRE MR images at arterial phase (Fig. 10(b) DNh). On the contrary, when the nodule evolves to a carcinoma, the decrease in the portal supply is progressively compensated by an increase in the arterial one, in a so-called neoangiogenesis process. Consequently, the tumor appears hyperintense at the arterial phase and hypointense at the portal one [Fig. 10(b)], when the concentration is higher in the normal tissue [Fig. 10(b)]. This enhancement is particularly strong for highly graded carcinoma (HCCp), for which the portal supply is completely absent in the tissue.

Although the capillarization phenomena tends to reduce the contrast enhancement at the arterial phase, as shown in the last 
paragraph, this enhancement is higher for the HCCp, for which, capillaries are almost impermeable. Indeed, on the graph of Fig. 8(b), we have seen that for a high arterial inflow, the concentration is high, whatever the permeability. This example illustrates rather well the difficulty of an interpretation from the single images, and the utility of a multiscale analysis.

\section{CONCLUSION}

The multiscale model of liver DCE-MRI presented in this paper has already shown good qualitative results regarding the coherence of contrast enhancements in pathological situations, especially in the case of a HCC. Furthermore, its ability to provide a multiscale analysis by coupling dynamic concentration curves to simulated MR images contributes to a better understanding of the physiological phenomena involved in the hepatocarcinogenesis. In the attempt to obtain more information on tumors, it is crucial for the model to describe the physiology and the dynamics of tissues that affect the contrast, as precisely and as realistically as possible. Here, the axially distributed model generates more realistic time distribution of the contrast agent in the liver parenchyma, than commonly used compartmental models, in which each compartment is a well-stirred tank. In addition, parameters used in the current model have a direct physiological, physical or geometrical meaning. However, an essential identification step is still to be achieved, before being able to provide a useful tool for the characterization and, eventually, for the diagnosis of the tumor. This problem might be partially overcome by experimental studies on animal models of HCC. In addition, a precise study of the evolution of other parameters related to the images, such as texture parameters, which has already shown an interest in tissue characterization, is foreseen. The determination of relationships between these features and the physiological parameters related to the model would thus help in solving the inverse problem, by representing bridges between observations on real images and the underlying physiological phenomena they reflect. In this aim, an initialization of the vascular model by vessel branches segmented from acquired images would allow the creation of a patient-specific model, more efficient in the characterization of the tumor.

\section{ACKNOWLEDGMENT}

The authors are particularly grateful to Prof. J. B. Bassingthwaighte for his precious advice regarding the modeling of transport phenomena in biological systems, and to Prof. J.-J. Bellanger for his interest in numerical aspects of this work.

\section{REFERENCES}

[1] J. Demongeot, J. Bézy-Wendling, J. Mattes, P. Haigron, N. Glade, and J. L. Coatrieux, "Multiscale modeling and imaging: The challenges of biocomplexity," Proc. IEEE, vol. 91, no. 10, p. 1723, Oct. 2003.

[2] F. X. Bosch, J. Ribes, R. Cleries, and M. Diaz, "Epidemiology of hepatocellular carcinoma clinics," Liver Disease, vol. 9, no. 2, pp. 191-211, 2005.

[3] H. B. El-Serag, J. Davila, and N. Petersen, "Dramatic rises in the incidence of hepatocellular carcinoma in the United States," in Proc. Digestive Disease Week 2003 Conf., Orlando, FL, 2003, pp. 17-23.
[4] D. V. Sahani and S. P. Kalva, "Imaging the liver," Oncologist, vol. 9, pp. 385-397, 2004.

[5] L. Caldeira and J. Sanches, "Liver tumor assessment with DCE-MRI," in Proc. 5th IEEE Int. Symp. Biomed. Imag.: From Nano to Macro, Paris, France, 2008, pp. 804-807.

[6] J. Bézy-Wendling and A. Bruno, "A 3-D dynamic model of vascular trees," J. Biol. Syst., vol. 7, no. 1, pp. 11-13, 1999.

[7] J. Bézy-Wendling and M. Kretowski, "Physiological modeling of tumor-affected renal circulation," Comput. Methods Progr. Biomed., vol. 91, no. 1, pp. 1-12, 2008.

[8] M. Kretowski, Y. Rolland, J. Bézy-Wendling, and J. L. Coatrieux, "Physiologically-based modeling for medical image analysis: Application to 3D vascular networks and CT scan modality," IEEE Trans. Med. Imag., vol. 22, no. 1, pp. 248-257, Jan. 2003.

[9] M. Kretowski, Y. Bézy-Wendling, and P. Coupé, "Simulation of biphasic CT findings in hepatic cellular carcinoma by a two-level physiological model," IEEE Trans. Biomed. Eng., vol. 54, no. 3, pp. 538-542, Mar. 2007.

[10] B. A. Lloyd, D. Szczerba, M. Rudin, and G. Svekely, "A computational framework for modelling solid tumor growth," Phil. Trans. R. Soc. A, vol. 366, pp. 3301-3318, 2008.

[11] M. Kretowski and J. Bézy-Wendling, "Modeling for medical image analysis: Framework and applications," in Medical Imaging Systems Technology, Analysis and Computational Methods, C. T. Leondes, Ed. Singapore: World Scientific, 2005, vol. I, pp. 1-32.

[12] W. Schreiner and P. F. Buxbaum, "Computer-optimization of vascular trees," IEEE Trans. Biomed. Eng., vol. 40, no. 5, pp. 482-491, May 1993.

[13] R. Karch, F. Neumann, M. Neumann, and W. Schreiner, "A three-dimensional model for arterial tree representation, generated by constrained constructive optimization," Comput. Biol. Med., vol. 29, pp. 19-38, 1999.

[14] M. Zamir and H. Chee, "Branching characteristics of human coronary arteries," Can. J. Physiol. Pharmacol., vol. 64, pp. 662-668, 1986.

[15] M. Kretowski, Y. Rolland, J. Bézy-Wendling, and J. L. Coatrieux, "Fast 3D modeling of vascular trees," Comput. Meth. Progr. Biomed., vol. 70, no. 2, pp. 129-136, 2003.

[16] M. Mescam, P. A. Eliat, C. Fauvel, J. D. De Certaines, and J. BézyWendling, "A physiologically-based pharmacokinetic model of vascular-extravascular exchanges during liver carcinogenesis: Application to MRI contrast agents," Contrast Media Molecular Imag., vol. 2, no. 5, pp. 215-228, 2007.

[17] F. E. Curry, "Mechanics and thermodynamics of transcapillary exchange," in Handbook of Physiology, The Cardivascular System. Microcirculation, Am. Physiol. Soc.. Bethesda, MD: , 1984, vol. IV, ch. 8, sec. 2, pt. 1, pp. 309-374.

[18] J. B. Bassingthwaighte, "A practical extension of hydrodynamic theory of porous transport for hydrophilic solutes," Microcircul., vol. 13, pp. 111-118, 2006.

[19] O. Kedem and A. Katchalsky, "Thermodynamic analysis of the permeability of biological membranes to non-electrolytes," Biochimica Biophysica Acta, vol. 27, pp. 229-246, 1958.

[20] O. Kedem and A. Katchalsky, "A physical interpretation of the phenomenological coefficients of membrane permeability," J. Gen. Physiol., vol. 45, pp. 143-179, 1961.

[21] C. S. Patlak, D. A. Goldstein, and J. F. Hoffman, "The flow of solute and sovent across a two-membrane system," J. Theoret. Biol., vol. 5, pp. 426-442, 1963.

[22] J. B. Bassingthwaighte, C. Y. Wang, and I. S. Chan, "Blood-tissue exchange via transport and transformation by capillary endothelial cells," Circul. Res., vol. 65, pp. 997-1020, 1989.

[23] C. A. Goresky, "A linear method for determining liver sinusoidal and extravascular volumes," Am. J. Physiol., vol. 204, pp. 626-640, 1963.

[24] H. Benoit-Cattin, G. Collewet, B. Belaroussi, H. Saint-Jalmes, and C. Odet, "The SIMRI project: A versatile and interactive MRI simulator," J. Magn. Res., vol. 173, pp. 97-115, 2005.

[25] O. Matsui, "Detection and characterization of small hepatocellular carcinoma," Clin. Gastroenterol. Hepatol., vol. 3, pp. S136-S140, 2005.

[26] F. Braet, D. Luo, I. Spector, D. Vermijlen, and E. Wisse, , I. M. Arias, J. L. Boyer, F. V. Chisari, N. Fausto, W. B. Jakoby, D. Scachter, and D. A. Shafritz, Eds., "Endothelial and pit cells," in The Liver: Biology and Pathobiology, 4th ed. Philadelphia, PA: Lippincott Williams Wilkins, 2001, pp. 437-453. 